rh 19 Revue d'histoire du XIXe siècle

Société d'histoire de la révolution de 1848 et des

révolutions du XIXe siècle

$62 \mid 2021$

Ancêtres

\title{
W. Caleb McDANIEL, Sweet Taste of Liberty: A True Story of Slavery and Restitution in America
}

\section{Soazig Villerbu}

\section{OpenEdition \\ Journals}

Édition électronique

URL : https://journals.openedition.org/rh19/7649

DOl : $10.4000 /$ rh 19.7649

ISSN : $1777-5329$

\section{Éditeur}

La Société de 1848

\section{Édition imprimée}

Date de publication : 20 juin 2021

Pagination : 263-265

ISSN : 1265-1354

Référence électronique

Soazig Villerbu, « W. Caleb McDANIEL, Sweet Taste of Liberty: A True Story of Slavery and Restitution in America ", Revue d'histoire du XIXe siècle [En ligne], 62 | 2021, mis en ligne le 17 août 2021, consulté le 26 août 2021. URL : http://journals.openedition.org/rh19/7649 ; DOI : https://doi.org/10.4000/ rh19.7649

Ce document a été généré automatiquement le 26 août 2021.

Tous droits réservés 


\title{
W. Caleb McDANIEL, Sweet Taste of Liberty: A True Story of Slavery and Restitution in America
}

\author{
Soazig Villerbu
}

\section{RÉFÉRENCE}

W. Caleb McDANIEL, Sweet Taste of Liberty: A True Story of Slavery and Restitution in America, New York, Oxford University Press, 2019, 288 p., $19 €$.

1 Après un ouvrage déjà primé relatif aux réseaux transatlantiques de l'abolitionniste William Lloyd Garrison, W. Caleb McDaniel publie ce nouveau livre récompensé par le prix Pulitzer (mais aussi le prix Avery O. Craven de l'Organization of American Historians) qui répond, comme souvent, à une demande sociale forte. Le prix a été en effet remis au nom d'une "recherche brillante au service d'une méditation sur le principe des réparations ${ }^{1}{ }^{\star}$ dont on voit bien que l'objectif est de poser encore et toujours les termes d'un débat public sur la mémoire de l'esclavage aux États-Unis. Cela dit, la question des réparations n'occupe finalement pas une place centrale dans l'ouvrage : certes l'introduction la pose en enjeu fort de l'écriture mais finalement elle n'intervient réellement que dans les dernières pages du livre, ce qui génère in fine une sorte de frustration. Elle pouvait pourtant s'appuyer sur de plus larges espaces historiographiques. De ce côté de l'Atlantique le projet ANR « Repairs » et sa nouvelle revue Esclavages et post-esclavages ${ }^{2}$, fait ainsi écho à la permanence du débat aux ÉtatsUnis et son aspect brûlant ces derniers temps.

2 Mais cela est sans doute avant tout dû au principe narratif retenu par McDaniel, qui à la fois s'inscrit dans des tendances lourdes de l'histoire aux États-Unis et répond clairement à l'objectif de toucher un plus large public que le strict milieu universitaire. Car l'auteur a une histoire à raconter: celle de Henrietta Wood, née esclave au Kentucky en 1818 ou 1820, dont le destin est, par la plume de McDaniel, entrecroisé 
avec l'itinéraire de Zebulon Ward, qui a participé à son enlèvement. L'intrigue est la suivante. Wood est d'abord une esclave du Haut-Sud, séparée enfant de sa famille, allant d'un propriétaire à un autre au gré de leurs difficultés financières, se retrouvant un temps à La Nouvelle-Orléans aux mains du marchand William Cirode, dont la faillite mène indirectement à son affranchissement en 1848 par la traversée de l'Ohio et à quelques années de vie libre à Cincinnati. Mais en 1853 elle est enlevée sur la rive nord du fleuve dans le cadre d'un trafic tout à fait banal sur cette frontière entre États libres et États esclavagistes, et renvoyée illégalement à sa condition servile originelle. Cette fois, elle connaît le Sud profond, les plantations de coton de la famille Branson autour de Natchez, puis durant la guerre de Sécession, la fuite du maître vers le Texas. La fin de la guerre dure pour elle des années, avant qu'elle puisse rentrer à Cincinnati avec son fils de père inconnu pour y renouer le fil de sa vie libre. C'est en travaillant en temps chez Myers, un avocat de Covington (dans le Kentucky, sur la rive sud de l'Ohio), qu'elle prend l'initiative de déposer plainte contre Zebulon Ward. Celui-ci, trafiquant d'esclaves, propriétaire de chevaux de course, avait fini par s'enrichir considérablement en assurant la gestion de différentes prisons privatisées par les États fédérés, et notamment en participant à la création d'un système carcéral sudiste qui relève d'une remise en esclavage des populations noires. Néanmoins la justice du Kentucky tranche en faveur de l'ancienne esclave : en 1879, le dossier est clos et Ward doit payer à Wood $2500 \$$ au titre de réparations pour l'avoir privée illégalement de liberté. On est loin des 20000 \$ que Wood réclamait mais le symbole est d'une force rare.

3 La recherche est effectivement d'un haut niveau : McDaniel a fait feu de tout bois pour reconstruire une vie d'esclave, ce qui est toujours difficile, comme pour éclairer celle de Ward, certes membre de l'élite sudiste mais qui n'a pas laissé de papiers personnels. De multiples fonds d'archives sont utilisés, de tous types, et la presse est mise à contribution. On peut malgré tout regretter une certaine légèreté dans le traitement de William Cirode : Guillaume, de son prénom de baptême, était arrivé de Nantes en 1816 et il est sans doute trop simple d'affirmer qu' « il appartenait à la diaspora en expansion venue de France napoléonienne et de Saint-Domingue » (p. 27). La formulation n'est pas d'une grande clarté et même Éric Saugera, qui a bien étudié la question, n'était pas si affirmatif que cela sur Cirode lui-même ${ }^{3}$; il est sans doute trop facile de déduire d'une migration en 1816 l'exode d'un partisan de Napoléon. Mais il eût fallu pour aller plus avant se pencher sur des sources francophones, alors que déjà McDaniel dans son premier opus traitait du monde radical atlantique en ne se fondant que sur des sources anglophones.

4 La bibliographie de son côté est impeccable. Ne connaître que des bribes de vie de Wood comme de Ward impose à McDaniel une contextualisation de tous les instants afin de reconstruire les environnements dans lesquels ces deux vies furent vécues faute de pouvoir les éclairer en détail. Logiquement le résultat est une suite d'exposés historiographiques parfaitement au point sur l'ensemble des questions touchant à l'esclavage et à l'histoire du Sud en général, même si McDaniel ne rentre pas suffisamment dans les débats. Ainsi lorsqu'il lui faut décrire le monde des plantations, il reprend pour l'essentiel les travaux récents de Walter Johnson, Calvin Schermhorn et Edward Baptist ${ }^{4}$. Or ce sont trois des historiens qui ont contribué ces derniers temps à relancer fortement le débat sur le lien entre capitalisme et esclavage, en faisant du second le parangon du premier et en usant pour ce faire d'arguments parfois contestables. Il n'est malheureusement pas trace de ces débats dans Sweet Taste of 
Liberty. Cela ne fait que mettre au clair ce qui est le souci de ce livre : on n'en saisit pas complètement le projet scientifique. Pourquoi exactement écrire cette histoire et de cette manière-là, voilà deux questions qui ne trouvent pas de réponse précise. Sur le fond, un lecteur ou une lectrice universitaire au fait de l'histoire de l'esclavage étatsunien n'y apprendra rien qu'il ne sait pas déjà. Il y trouvera un bon résumé de points importants, le tout enrobant une intrigue parfaitement déroulée et écrite d'une plume alerte. On peut presque supposer que c'est cela que vient couronner le prix Pulitzer : une histoire bien écrite, pour un grand public éclairé qu'il tiendra au fait des évolutions historiographiques en le gâtant d'une histoire qui le fera réfléchir à la société contemporaine. L'objectif est louable, même si on pouvait attendre autre chose d'une telle étude de cas - mais ce livre est-il même conçu comme une étude de cas ? Ou s'agitil de microhistoire vulgarisée perçue uniquement comme dernière solution pour attirer un lectorat vers les ouvrages d'histoire scientifiques ${ }^{5}$ ?

\section{NOTES}

1. The Pulitzer Prizes [https://www.pulitzer.org/prize-winners-by-category/220] (consulté en avril 2021).

2. Projet ANR "Repairs" [https://repairs.hypotheses.org] ; Revue Esclavages et post-esclavages [https://journals.openedition.org/slaveries/] (sites consultés en avril 2021).

3. Eric Saugera, Reborn in America: French Exiles and Refugees in the United States and the Vine and Olive Adventure, 1815-1865, Tuscaloosa, University of Alabama Press, 2011.

4. Walter Johnson, The River of Dark Dreams: Slavery and Empire in the Cotton Kingdom, Cambridge, The Belknap Press of Harvard University Press, 2013 ; Edward Baptist, The Half had Never Been Told: Slavery and the Making of American Capitalism, New York, Basic Books, 2014 ; Calvin Schermerhorn, The Business of Slavery and the Rise of American Capitalism, New Haven, Yale University Press, 2015.

5. Thomas Robisheaux, Thomas V. Cohenand, István M. Szijártó (eds.), “Microhistory Today: A Roundtable Discussion”, Journal of Medieval and Early Modern Studies, vol. 47, 2017/1, p. 7-52. 